\title{
Effective Concentration and Detection of Cryptosporidium, Giardia, and the Microsporidia from Environmental Matrices
}

\author{
Joseph A. Moss, ${ }^{1}$ John Gordy, ${ }^{2}$ and Richard A. Snyder ${ }^{1}$ \\ ${ }^{1}$ Center for Environmental Diagnostics and Bioremediation, University of West Florida, 11,000 University Parkway, \\ Building 58, Room 70, Pensacola, FL 32514, USA \\ ${ }^{2}$ Water Quality Laboratory, City of Tampa Water Department, 7125 North 30th Street, Tampa, FL 33610, USA \\ Correspondence should be addressed to Joseph A. Moss; jmoss@uwf.edu
}

Received 31 May 2014; Revised 11 August 2014; Accepted 21 August 2014; Published 10 September 2014

Academic Editor: Nongnuch Vanittanakom

Copyright (C) 2014 Joseph A. Moss et al. This is an open access article distributed under the Creative Commons Attribution License, which permits unrestricted use, distribution, and reproduction in any medium, provided the original work is properly cited.

\begin{abstract}
Cryptosporidium spp., Giardia spp., and members of Microsporidia are enteropathogenic parasites of humans and animals, producing asymptomatic to severe intestinal infections. To circumvent various impediments associated with current detection methods, we tested a method providing multistage purification and separation in a single, confined step. Standard real-time PCR was used as a detection method. Samples spiked with C. parvum and G. intestinalis were split for comparison to standard Method 1623. Results were equivalent to immunomagnetic procedures for Cryptosporidium, and Giardia. Overall percent recovery for Cryptosporidium with Method 1623 averaged 26.89\% (std 21.44\%; $\min =0 \%$; $\max =73 \%$ ) and was similar but less variable for qPCR method at an estimated average of 27.67 ( $\operatorname{std} 17.65 \%$; $\min =5 \%$; $\max =63 \%$ ). For Giardia, Method 1623 had an overall average recovery of $27.11 \%$ (std $17.98 \%$; $\min =1 \%$; $\max =58 \%$ ), while multistage purification and qPCR had an estimated lower overall recovery at $18.58 \%$ (std $13.95 \%$; $\min =0 \%$; $\max =35 \%$ ). Microsporidia were also readily detected with an estimated recovery of $46.81 \%$ overall (std $17.66 \%$; $\min =18 \%$; $\max =70 \%$ ) for E. intestinalis and $38.90 \%$ (std $14.36 \%$; $\min =13 \% ; \max =62 \%)$ for $E$. bieneusi.
\end{abstract}

\section{Introduction}

Cryptosporidium, Giardia, and Microsporidia are enteropathogenic parasites of humans and animals, producing asymptomatic to severe intestinal infections $[1,2]$. Detection of these pathogens continues to be of great interest for public health, and direct detection monitoring is warranted given a poor correlation with standard fecal pollution indicators [3]. Currently, the U.S. and several European nations mandate the use of combined immunomagnetic and microscopy-based (IMS) procedures (i.e., IT rule; Method 1623) for monitoring surface and drinking waters for Cryptosporidium and Giardia. These methods are hampered by cost and their time consuming nature [4] and require specific expertise to distinguish between human and animal pathogenic Cryptosporidium and Giardia species [5]. Problems also arise with false positive and negative results [6] and poor dissociation of oocysts from magnetic beads in the purification step [7]. These methods are specific only to Cryptosporidium and Giardia, and though Microsporidia and other waterborne pathogens are listed in various contaminate lists, no cost and time-effective method for their detection exists.

It is well known that molecular techniques have been developed that are more effective than immunofluorescence microscopy in detecting specific pathogens [8]. Notably, recreational and environmental waters, such as surface waters and areas impacted by sewage outfalls present complex sample matrices known to contain numerous organic and inorganic dissolved and particulate substances that can affect sample collection and purification as well as having the potential to inhibit PCR reactions [6]. Many current molecular-based methods rely upon IMS to alleviate these problems. The IMS technique is not without difficulties; highly turbid samples affect pathogen recovery [9] resulting in either low and/or variable rates [7]. In addition, nonspecific binding of antibodies in complex samples appears to be 
a factor limiting detection sensitivities. Therefore, affordable alternative methods are needed to help bolster pathogen detection in water matrices.

To circumvent problems associated with IMS, a basic method combining filter dissolution and separation of targets in a centrifugation device (CFD) was evaluated. The procedure was coupled with downstream qPCR for a rapid, cost-effective approach to detecting these pathogens. This report describes the procedure and presents data regarding sensitivity and efficacy in a comparative evaluation to use of IMS microscopy performed in a NELAC certified laboratory.

\section{Materials and Methods}

2.1. Pathogens. Inactivated Cryptosporidium parvum oocysts (CpAZ strain) and $G$. intestinalis (H3 isolate, assemblage B) cysts (gamma-irradiated) used for spiking suspension in all trials (Method 1623 and qPCR) and standards (qPCR). Pathogens were flow cytometry-sorted and suspended in $0.75 \mathrm{~mL}$ of reagent water with $0.01 \%$ Tween 20 , which had been inactivated and preserved (Accuspike-IR; Waterborne Inc., LA). Encephalitozoon intestinalis were supplied as purified suspensions in phosphate-buffered (PBS) solution $(1 \times$ $10^{6}$ spores; Didier isolate; P103I) and Enterocytozoon bieneusi spores were supplied as unpurified suspensions (Waterborne Inc; New Orleans, LA). All oocysts, spores, and cysts were purchased 3-4 weeks prior to trials and stored at $4^{\circ} \mathrm{C}$. All parasites are evaluated for general quality and intactness under differential interference contrast microscopy.

2.2. Comparative Trials. For a comparative evaluation with IMS-microscopy (Method 1623; [10]), bulk volumes of water of varying turbidity ( $<1$ to 54 NTUs) were divided into separate 20-25 L volumes and spiked with known quantities of C. parvum oocysts and G. intestinalis cysts (Accu-spike; Waterborne Inc. New Orleans, LA). To standardize turbidity treatments and to ensure the absence of pathogens in water samples prior to spiking, water sample volumes having turbidities $>1$ were created by adding a purified loam of soil to Milli-Q purified water (18 M $\Omega$; Millipore, Bedford, MA). Soil was autoclaved twice at $121^{\circ} \mathrm{C}$ for $20 \mathrm{~min}$ at $24 \mathrm{hr}$ intervals prior to mixing with water. Water used for spiking with turbidity standards $<1$ NTUs (potable water) was provided at the City of Tampa Water Department (Biol. Dept).

Water of varying turbidities was divided into separate polycarbonate carboys (20-25 L). In each of 2 trials, sample water of 3 turbidities (3 replicates/turbidity) was spiked with 100 (oo)cysts each of C. parvum and G. intestinalis. Prior to spiking, representative aliquots from carboys were tested for turbidity using a turbidimeter $(2100 \mathrm{~N}, \mathrm{Hach})$. Carboys were spiked with gamma-irradiated C. parvum oocysts and $G$. intestinalis cysts according to the manufacturer's instructions (AccuSpike; Waterborne Inc, LA). Additionally, carboys were spiked with predetermined counts of E. intestinalis and E. bieneusi for evaluation of separation via the CFD and detection using qPCR. Estimates for spiking suspensions and for $\mathrm{qPCR}$ standard curves were done via hemocytometer counts. The standard suspensions of Microsporidia were prepared 1 week prior to each trial. Method blanks, in which no pathogens were added, were included in trial 1.

Spiked samples evaluated using Method 1623 were filtered through FiltaMax filters (Idexx) with flow volumes recorded using a digital rate meter (model 220/101-8T Flo-Sensor; McMillan). Sample volumes evaluated using filter dissolution combined with qPCR were collected on $142 \mathrm{~mm}$ mixed cellulose ester (MCE; 1.2 um porosity; Millipore) membranes using a filter tower ( $5 \mathrm{~mm} \mathrm{Hg}$ vacuum). MCE filters were folded, placed into zip-lock bags, and labeled accordingly. Filters were transported to UWF on ice and processed within 48 hrs.

2.3. Environmental Samples. Environmental samples were collected from the Pensacola and the Tampa Bay region. A few additional samples were collected from rivers and waste water treatment facilities (WWTF) in St. Petersburg and Auburndale, Florida. Samples from the Pensacola region were collected into polycarbonate carboys and transported back to the lab for filtering and processing. Unused portions of samples processed by routine testing at the City of Tampa Water facility by Method 1623 were used for evaluation via qPCR. Unused volumes were filtered onto 1.2 $\mu \mathrm{m}$ MCE filters which were shipped overnight to UWF. The volumes were not equal but provided additional testing material.

2.4. Method 1623. Samples were processed as described for the IMS microscopy method (Method 1623; [10]) at the Water Quality Laboratory at the City of Tampa Water Department. The Dynal IMS procedure (Dynabeads G/C combo IMS kit; Dynal A.S., Oslo, Norway) was performed according to the manufacturer's instruction. Adhesion slide preparations were immunostained with fluorescein isothiocyanate (FITC) labeled antibody (Meridian Biosciences) following manufacturer's instructions and stained with $4^{\prime}, 6$-diamidino-2phenylindole (DAPI) $0.001 \mathrm{mg} / \mathrm{mL}$ (Sigma-Aldrich) according to Method 1623. Enumeration of oocysts and cysts was accomplished using an Olympus BX60 microscope with magnifications of $200 \mathrm{x}-1000 \mathrm{x}$ for the examination of immunofluorescence (FA), DAPI staining characteristics, and differential interference contrast (DIC) microscopy according to Method 1623.

2.5. Centrifugation-Filtration Purification. The study utilized a multitiered centrifugation-filtration (CFD; GenIUL Terrassa, Spain) device (Figure 1) which consists of interlocking (vapor-tight) sections for separation of large particulates and for collection of target organisms. Prior to filter dissolution, a $47 \mathrm{~mm}$ solvent resistant "retention" filter $(2 \mu \mathrm{m}$; Nuclepore Track-Etched, Whatman) was placed upon the mesh screen of the lower section (Section 3, Figure 1(a)) to collect target pathogens; $47 \mathrm{~mm}$ prescreens $(20 \mu \mathrm{m}$; Nytex $)$ were placed upon mesh supports to remove larger particulates.

For dissolution, the MCE filters were folded using sterile forceps and inserted into the primary chamber (Section 1; Figure 1(a)) of the CFD. This was followed by addition of $\sim 30 \mathrm{~mL}$ acetone $(95-100 \%)$ and $\sim 25$ zirconia/silica beads (2.3 mm; BioSpec Inc.). The lid was secured on the primary 


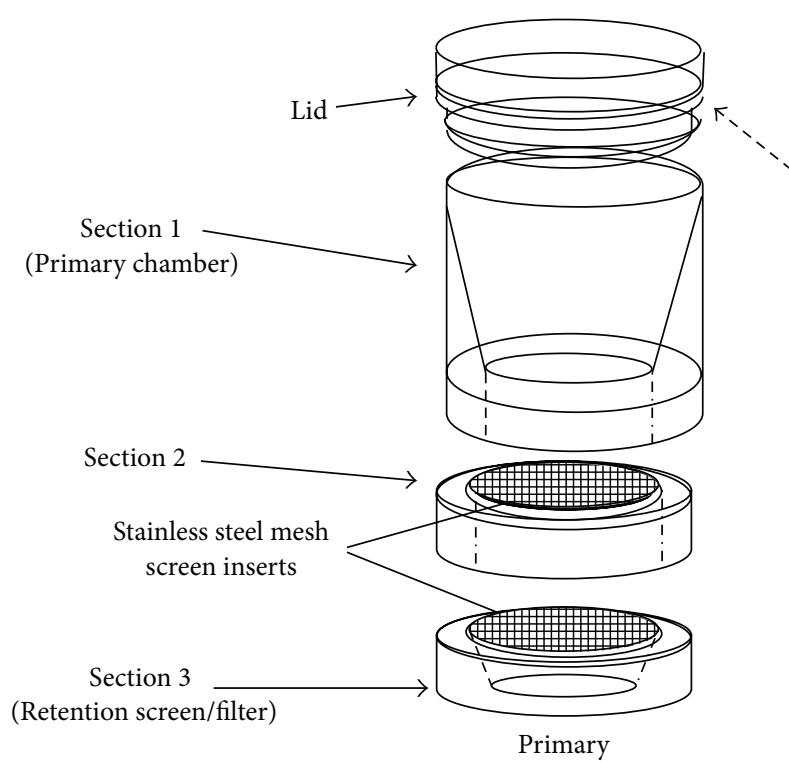

(a)

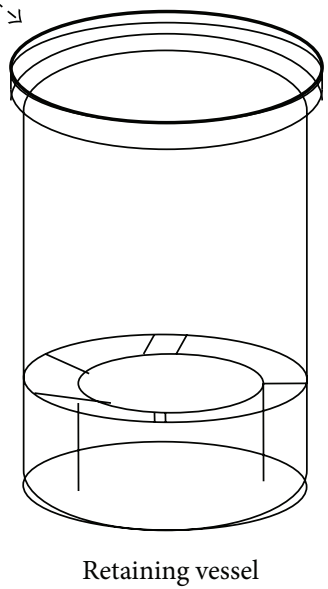

(b)

FIGURE 1: Illustration of centrifugation-filtration device (CFD) utilized.

apparatus which was then fastened into its retaining vessel. The unit was thoroughly agitated (hand shaking; $~ 1 \mathrm{~min}$ ) and centrifuged at $\sim 2000-3600 \mathrm{rcf}$ for 3-5 min (Sorvall RC$5 \mathrm{~B} / \mathrm{C}$ with a GSA rotor; DuPont). The primary unit was then removed from its retaining vessel and flow-through discarded. Two additional wash steps using acetone (100\%) and centrifugation (2000-3600 rcf for 2-3 min) were performed. Following completion of wash steps, the primary unit was inspected to ensure that no residual dissolution fluid remained on the retention filter. When necessary, residual fluid was removed via additional centrifugation and/or by application of vacuum to the bottom of the unit by a filtration manifold.

2.6. gDNA Extraction. Retention filters were removed from CFD units with sterile forceps and inserted into separate $2 \mathrm{~mL}$, bead-beading tubes (Powersoil; MoBio). Lysis buffer was added and filters were subjected to 3 freeze-thaw (liquid nitrogen $/ 65^{\circ} \mathrm{C}$ ) cycles. Tubes were agitated using a FastPrep (ThermoSavant) or a PowerLyzer homogenizer (MoBio) using 45 second bursts at setting 4.5 and S3500, respectively. Genomic DNA extraction was done according to the manufacturer's instructions and stored at $-20^{\circ} \mathrm{C}$.

2.7. Amplification and Detection. Primer-probe sets which were used to detect $G$. intestinalis cysts and $C$. parvum oocysts consisted of those targeting the $\beta$-Giardin gene and the gene for the Cryptosporidium oocyst outer wall protein (COWP; [11]). PCRs were performed in $15 \mu \mathrm{L}$ volumes containing $3.0 \mathrm{mM} \mathrm{MgCl}_{2}$, PCR buffer (FastTaq 10x/Green), $0.50 \mu \mathrm{M}$ each primer, $0.04 \mathrm{U}$ of FastStart Taq DNA polymerase (Roche), 0.2 mM PCR Nucleotide MixPlus (Roche), 0.025\% bovine serum albumin (Sigma, St. Louis, Mo.), and $0.3 \mu \mathrm{M}$ of each hybridization probe. Cycling conditions were consistent with those of Guy et al. 2003. Fluorescence was measured at the end of each cycle. For detection of E. bieneusi spores, primers, and hybridization probe targeting the ITS region of the $18 \mathrm{~S}$ rRNA gene were utilized [12]. Primers and probe used for detection of Encephalitozoon spp. (pan-Encephalitozoon) spores were from Wolk et al. 2002 [13]. Reactions for detection of E. bieneusi and Encephalitozoon spp. were performed separately in $15 \mu \mathrm{L}$ total volumes containing $3.0 \mathrm{mM} \mathrm{MgCl}$ (Roche), PCR buffer (FastTaq 10x/Green), $0.40 \mu \mathrm{M}$ each primer, 0.04 U of FastStart Taq DNA polymerase (Roche)/l, $0.2 \mathrm{mM}$ PCR Nucleotide MixPlus (Roche), $0.025 \%$ bovine serum albumin (Sigma, St. Louis, Mo.), and $0.3 \mu \mathrm{M}$ of each hybridization probe. Thermal conditions for both reactions consisted of $5 \mathrm{~min}$ at $95^{\circ} \mathrm{C}$ followed by 50 cycles of $15 \mathrm{~s}$ at $95^{\circ} \mathrm{C}$, $30 \mathrm{~s}$ at $60^{\circ} \mathrm{C}$, and $30 \mathrm{~s}$ at $72^{\circ} \mathrm{C}$. Fluorescence was measured at annealing step of each cycle.

A minimum of 8-10 replicate reactions were used for all samples and results pooled. To ensure purity of reagents, "no template controls" (NTCs) were run with every PCR reaction series. Standard suspensions containing 100 and 500 cysts and oocysts and 1000 and 5000 spores were used to develop standard curves. Standards were subjected to gDNA extraction methodology as stated previously. The projected number of pathogens was calculated based on the portion of gDNA analyzed using the following formula: number of pathogens (i.e., oocysts, spores) in each sample $=$ (number of pathogens calculated $) \times($ total gDNA extraction volume $) /($ total volume of gDNA analyzed). All runs and data analysis were performed using Rotor-Gene 3000 (Corbett) real-time PCR machines and software. Pathogen presence and species identification in unknowns were verified using electrophoresis on $2 \%$ agarose gels and subsequent sequencing (Big Dye; V1.1; ABI 3100 


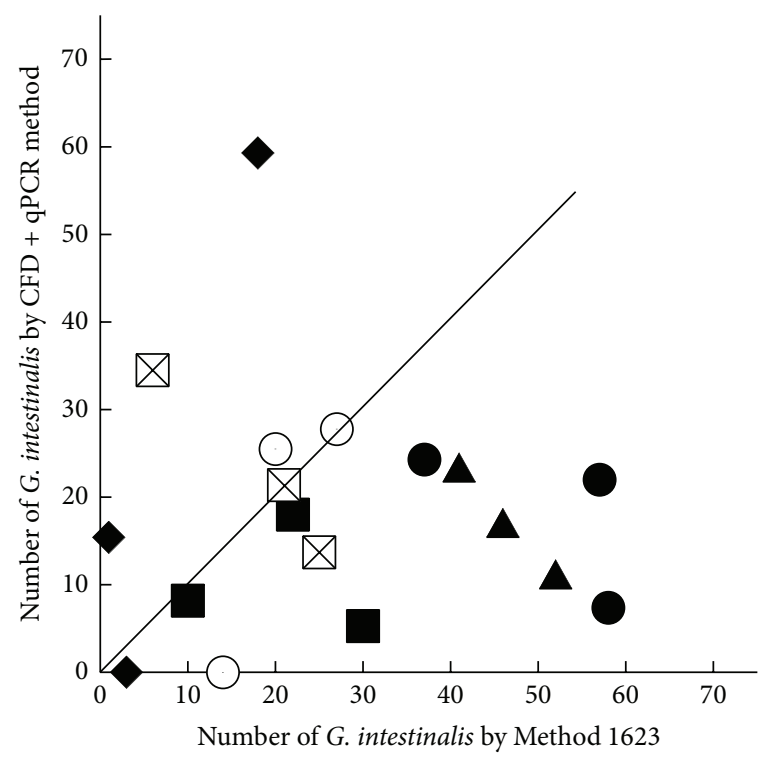

(a)

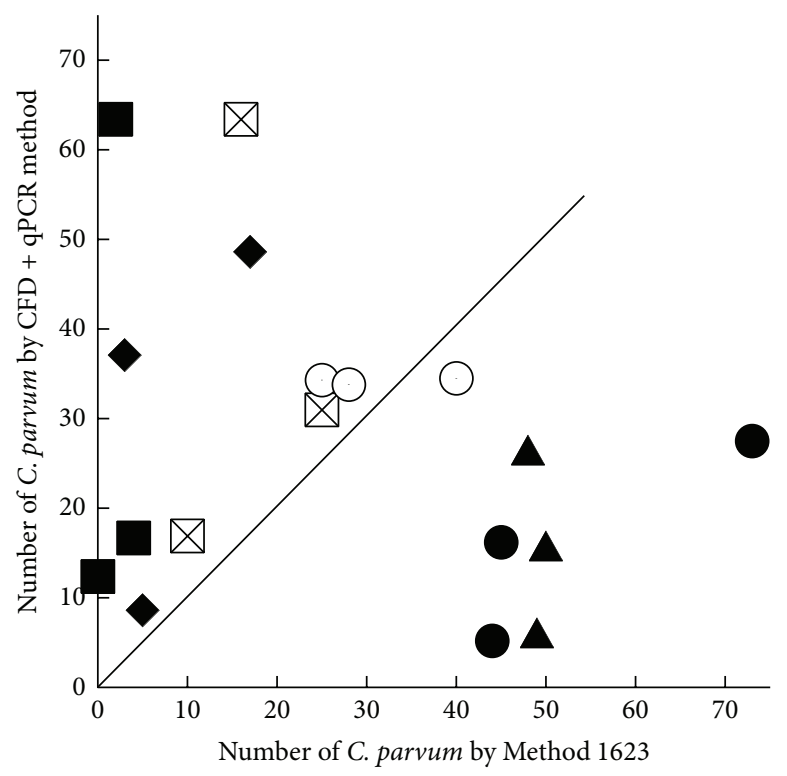

(b)

Figure 2: A comparison of split sample analysis at various turbidity amounts by Method 1623 and the CFD + qPCR method for Giardia intestinalis (a) and Cryptosporidium parvum (b). The solid lines represent a 1:1 correspondence of results. Solid circles: tap water 0.2 NTU, open circles: tap water 0.4 NTUs, x-squares: 1.1 to 1.3 NTUs, triangles: 3.8 NTUs, squares: 7.1 NTUs, and diamonds: 54 NTUs.

sequencer) according to manufacturer's instructions. Data were aligned against existing sequences for known pathogens (BLAST; NCBI).

\section{Results}

Using inactivated (gamma-irradiated) C. parvum oocysts and $G$. intestinalis cysts, parallel processing of split samples directly compared Method 1623 and qPCR. Data for the methods comparison are listed and presented (Table 1; Figure 2). Overall percent recovery for Cryptosporidium with Method 1623 averaged $26.89 \%$ (std 21.44\%; $\min =0 \%$; $\max =$ $73 \%$ ) and was similar but less variable for the CFD + qPCR method at an average of 27.67 (std 17.65\%; $\min =5 \%$; $\max =$ 63\%). For Giardia, Method 1623 had an overall average recovery of $27.11 \%$ ( std $17.98 \%$; $\min =1 \%$; $\max =58 \%$ ), while the CFD + qPCR had a lower overall recovery at $18.58 \%$ (std $13.95 \%$; $\min =0 \%$; $\max =35 \%$ ).

Microsporidian spores, E. intestinalis, and E. bieneusi spores were added to samples as well as $C$. parvum and $G$. intestinalis. The estimated number of $E$. intestinalis and $E$. bieneusi spores detected using CFD + qPCR and associated percent recovery for each sample are listed (Table 1). The percent recovery for the Microsporidia by qPCR was $46.81 \%$ overall (std $17.66 \% ; \min =18 \%$; $\max =70 \%$ ) for E. intestinalis and $38.90 \%$ ( std $14.36 \%$; $\min =13 \% ; \max =62 \%$ ) for $E$. bieneusi (Table 1). Use of two $142 \mathrm{~mm} \mathrm{MCE} \mathrm{filters} \mathrm{was} \mathrm{nec-}$ essary for samples having a turbidity of 54 NTUs. Additional agitation time ( $2 \mathrm{~min})$ was necessary to allow for complete dissolution of the filters as well as supplementary rinse steps (2-3 times) for removing additional cellulose.
A decline in the ability of Method 1623 to detect Cryptosporidium and Giardia cysts was observed with increasing turbidity, while the qPCR method was less affected by turbidity for detection of Cryptosporidium and Giardia (Figure 3) and E. intestinalis and E. bieneusi (Figure 4).

Data from qPCR using samples volumes left over after employment of Method 1623 is presented in Table 2. Use of qPCR enabled detection of Giardia intestinalis and Microsporidian pathogens in $80 \%$ of the environmental samples evaluated. Cryptosporidium numbers fell below detection thresholds. The majority of pathogens detected were primarily Microsporidia, with E. hellem in the majority of environmental and waste water samples.

\section{Discussion}

Finding the origin of fecal pollution is paramount in assessing associated health risks. However, microbial source tracking (MST) is often expensive, time-consuming, and intimidating to those who need it [14]. Most approaches are not logistically feasible to real-world situations and difficult to gauge for their effectiveness due to the fact there is no regulatory method for making comparisons [15]. This is particularly the case for PCR-based methodologies designed for detection, verification, and relative quantification of pathogens in environmental samples. MST methods need to be chosen using consideration of cost, reproducibility, discriminatory power, ease of interpretation, and ease of performance $[16$, 17]. Limitations of microscopy, including slow analysis time and inability to differentiate pathogen species and strains, have been rectified by molecular-based methodologies which 


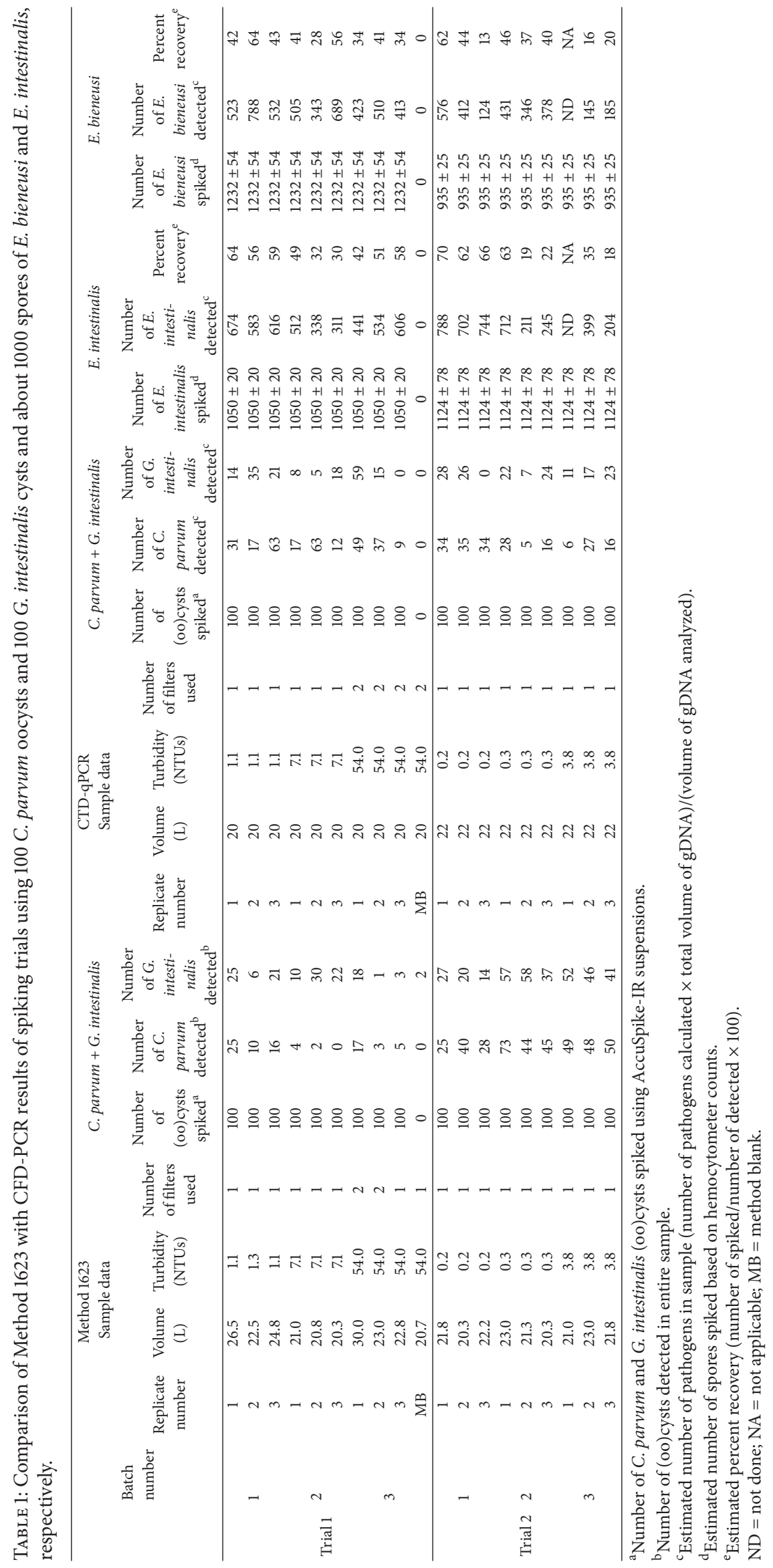




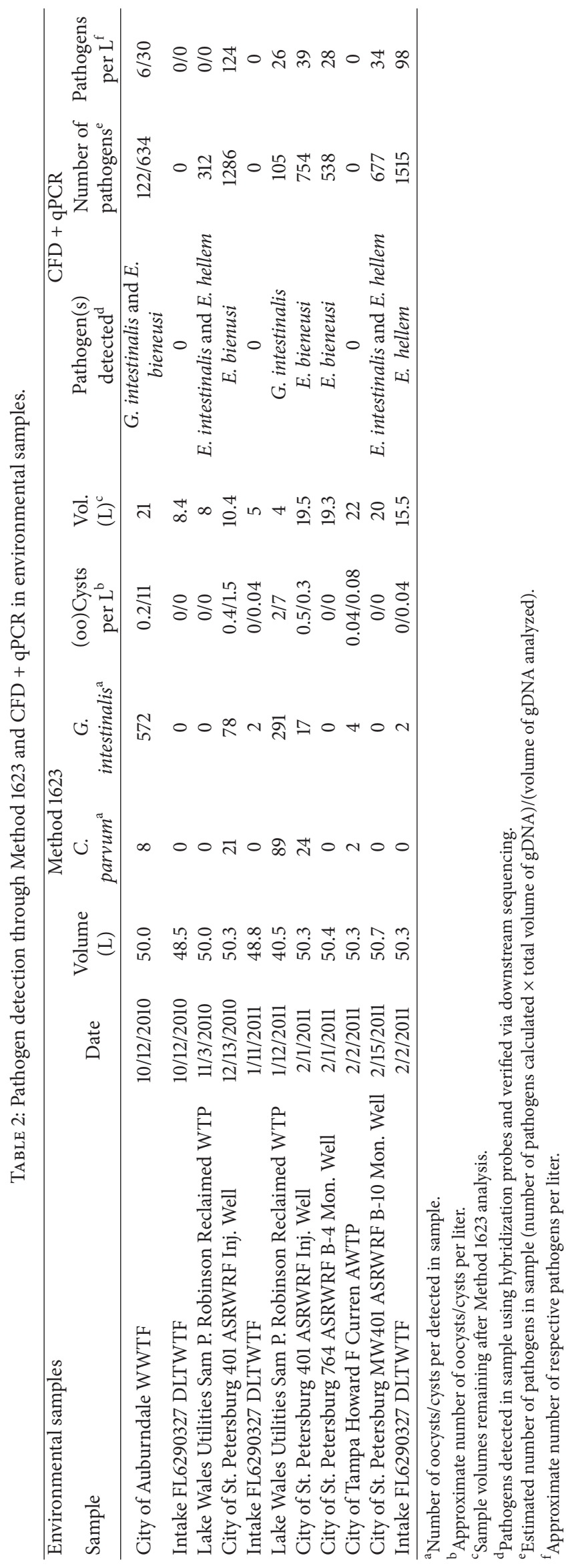




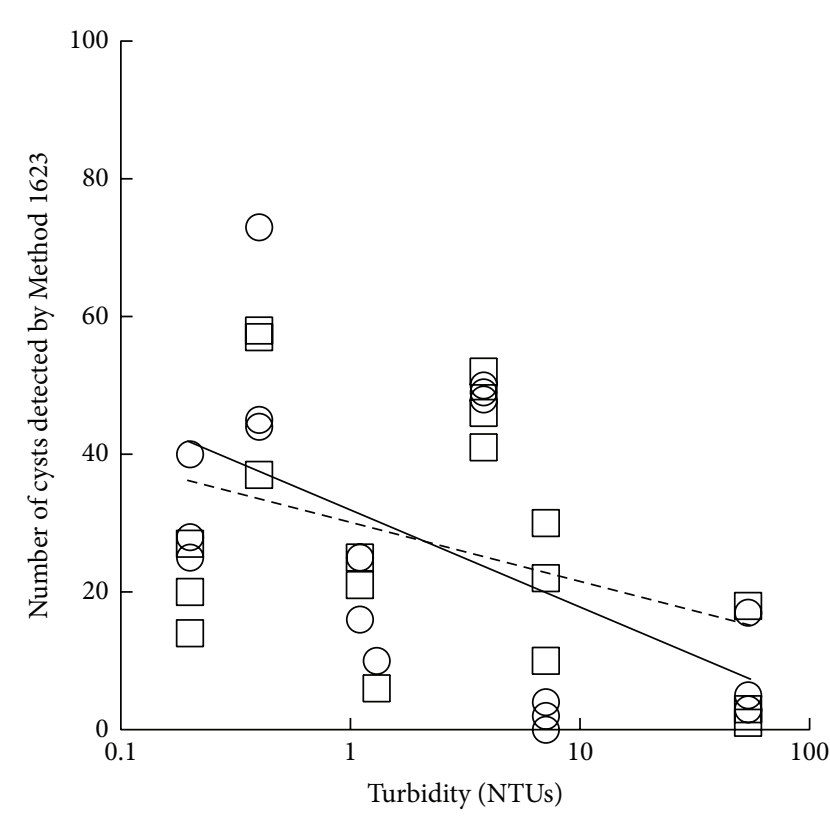

$\bigcirc$ Number of C. parvum by Method 1623 - Number of G. intestinalis by Method 1623

(a)

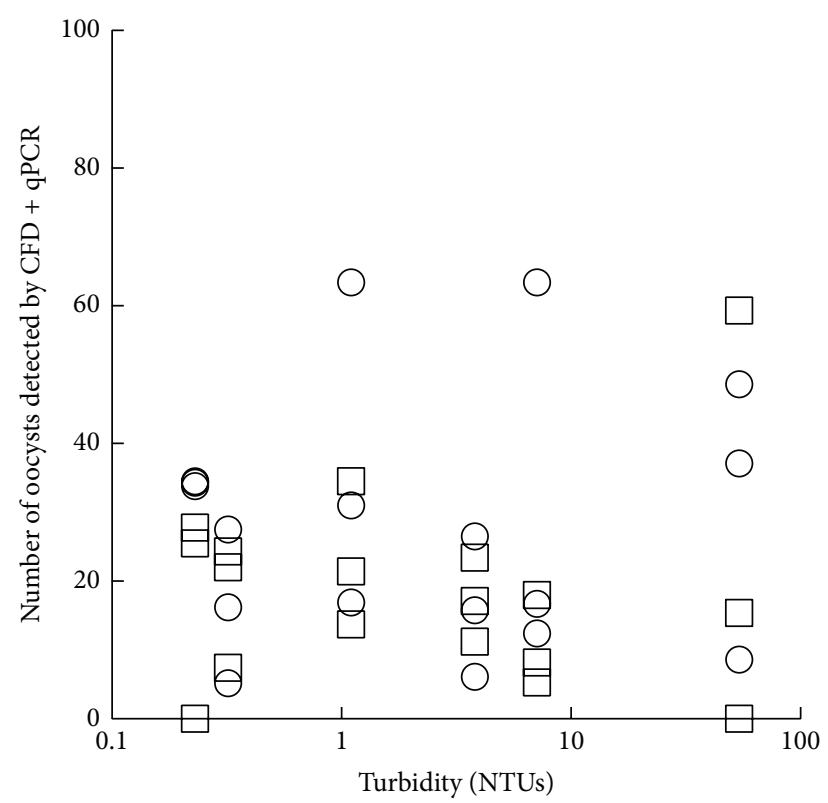

Number of C. parvum by CFD + qPCR Number of $G$. intestinalis by CFD + qPCR

(b)

FIGURE 3: The effect of increasing turbidity in samples on the performance of Method 1623 (a) and the CFD + qPCR method (b) for both Giardia intestinalis and Cryptosporidium parvum.

have also shown higher sensitivity than Method 1623 and the United Kingdom regulatory method $[18,19]$.

In the event of large outbreaks, analysis of numerous field samples to provide spatial and temporal assessments is necessary [20]. Arguably, the high cost (>\$400/sample; EPA-certified labs) associated with IMS-microscopy based detection prohibits such use and constrains from implementing routine monitoring. Costs incurred in this study were approximately $\$ 40 /$ sample and include costs for filters, gDNA extraction, and qPCR reagents and hybridization probes. The CFD methodology worked at a fraction of the cost (12\%) and time (6 times faster), based on evaluation of membrane filtration and IMS methodologies ([21]; this study). The methodology was shown to be effective in detection of pathogens present at moderate concentrations ( $\geq 5$ (oo)cysts/L) or higher. Isolation of pathogens present in the environments at lower concentrations may still prove to be inconstant, though the specificity of qPCR is high with low time consumption in contrast to visual methods. Additionally, the methodology used herein may aid in detection of various pathogens as commercial IMS kits do not permit collective isolation of multiple pathogens from single samples and have not been developed for other known pathogens (i.e., Cyclospora cayetanensis). Regardless, a combination of molecular and microscopy-based analyses should ultimately increase effectiveness of waterborne pathogen detection.

The purification approach in this study is an adaptation of the cellulose-acetate filter dissolution, having previous reported rates of recovery ranging from $70-79 \%$ for the recovery of Cryptosporidium spp. oocysts [22, 23]. Mean recoveries of $50.2 \%$ for Cryptosporidium oocysts and of $63.1 \%$ for Giardia cysts have also been reported [24]. The methodology has been used with microscopy [25] as well as PCR [26] and fluorescent in situ hybridization (FISH) [27]. However, losses have been attributed to the multiple centrifuge and aspiration steps, as well as the occurrence of a hardened pellet which made the process of detection of pathogens more difficult. These steps have been circumvented in this study, reducing the process to one combined step and bypassing the need for aspirating and pelleting of sample material and associated solvent vapor exposure.

Concerns over providing information on the viability and infectivity of protozoan pathogens have been made $[24,28]$. However, C. parvum oocysts were reported to be viable in mice following use of the methodology [29]. These issues may be addressed through use of propidium monoazide prior to acetone dissolution and PCR [30]. In any case, continual detection of a high concentration of pathogens in a sample despite capacity to confirm pathogenicity or viability should be of concern.

The multiplex primer-probe set [11] enabled evaluation of the CFD methodology and allowed for simultaneous amplification of both targets within a single reaction tube. Detection of $\sim 5$ (oo)cysts/L in water samples having turbidities of 3.8-54 NTUs was achievable; providing detection sensitivities which fall into acceptable limits with respect to reported ID-50s of $G$. intestinalis (25-100 cysts; [31, 32]) and C. parvum (10-132 oocysts, [33-35]) and estimates of average consumption of recreational water while bathing $(\sim 79.7 \mathrm{~mL}$, [36]; 50 mL, [37]). 


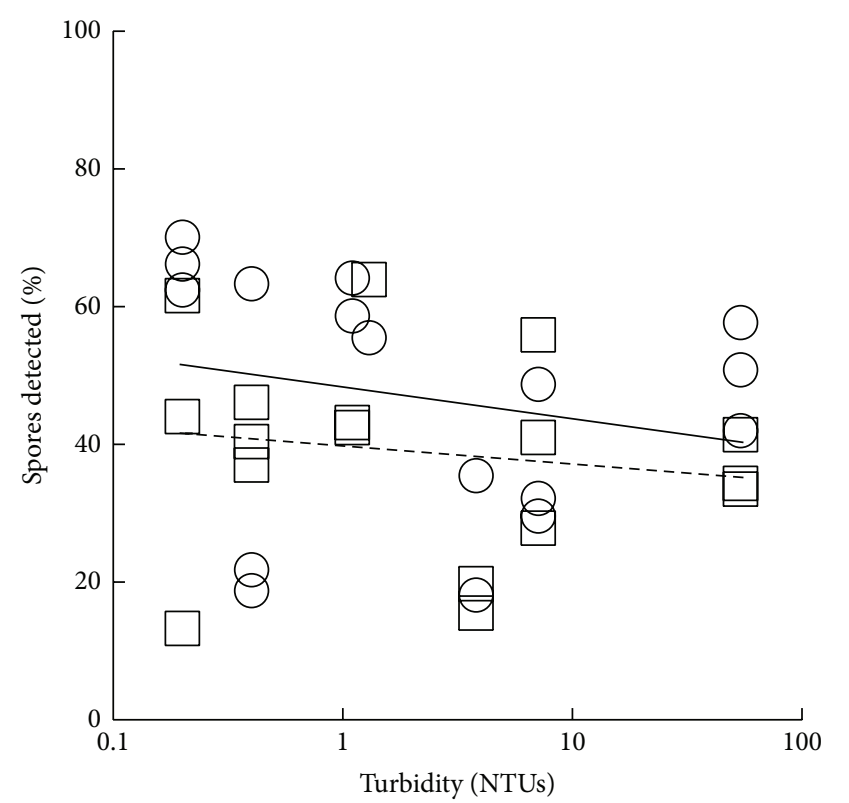

$\odot$ E. intestinalis

FIGURE 4: Detection efficiency of E. intestinalis and E. bieneusi by CFD + qPCR with increasing turbidity.

The methodology was successful in detection of pathogens in the spiked samples as well as in environmental samples. C. parvum was detected in a sample of raw sewage using qPCR, and other samples for sewage and sewage treatment plant effluents were positive for Microsporidia by qPCR. E. bieneusi was detected in a few of the samples collected from various freshwater sources. This pathogen is prevalent throughout the world and is found in a wide variety of hosts including pigs, humans, and other mammals $[38,39]$. Their presence, associated with waterborne outbreaks and also with recreational and river water, is continually being documented [36].

The number of samples and analytical replicates affects detection of pathogens in water by both microscopy and PCR [20]. A minimum of 10 replicate $\mathrm{qPCR}$ reactions were pooled to improve analytical sensitivity by accounting for the probability of missing target sequences in the aliquots taken from gDNA extract solutions [40]. Increasing the number of PCR reactions increases sensitivity. Various additional primer-probe combinations directed at detection of Cryptosporidium [41] and Giardia [42] currently exist, and certain primer-probe combinations may prove to work better. More importantly, recent advances like digital-droplet PCR should ultimately improve overall detection sensitivity. Reduction from $20 \mu \mathrm{m}$ to $12-15 \mu \mathrm{m}$ prescreens may improve the overall performance of the method. Nytex was used since, at the time, no stainless steel mesh prescreen was available. Additionally, gamma-irradiated, flow cytometry-sorted C. parvum and G. intestinalis suspensions (AccuSpike-IR, Waterborne Inc) were used in order to provide precision with regard to matrix spiking but also to provide a degree of safety during the development and evaluation of the assay. The effect of the gamma-irradiation on the performance of PCR is an unknown factor.

\section{Conclusion}

This report has demonstrated the capability of combining filter dissolution with qPCR for the direct detection of human enteric pathogens with results that are comparable to IMS microscopy, at substantial reductions in time and expense. Assay times were roughly 3-3.5 hr (1 technician/thermocycler, 1-6 samples) from initial filtration of samples to completion of qPCR. The method was straightforward and minimized sample manipulation.

\section{Conflict of Interests}

The authors declare that there is no conflict of interests regarding the publication of this paper.

\section{Acknowledgments}

This study was funded in part by the New Florida State University Research Assistance Grant. The authors thank Will Fitzpatrick and Jake Ziglioli for their help and expertise.

\section{References}

[1] E. S. Didier, "Microsporidiosis: an emerging and opportunistic infection in humans and animals," Acta Tropica, vol. 94, no. 1, pp. 61-76, 2005.

[2] L. Xiao and R. Fayer, "Molecular characterisation of species and genotypes of Cryptosporidium and Giardia and assessment of zoonotic transmission," International Journal for Parasitology, vol. 38, no. 11, pp. 1239-1255, 2008.

[3] V. J. Harwood, A. D. Levine, T. M. Scott et al., "Validity of the indicator organism paradigm for pathogen reduction in reclaimed water and public health protection," Applied and Environmental Microbiology, vol. 71, no. 6, pp. 3163-3170, 2005.

[4] J. M. Weintraub, "Improving Cryptosporidium testing methods: a public health perspective," Journal of Water and Health, vol. 4, no. 1, pp. 23-26, 2006.

[5] M. J. Allen, J. L. Clancy, and E. W. Rice, "Plain, hard truth about pathogen monitoring," Journal of the American Water Works Association, vol. 92, no. 9, pp. 64-76, 2000.

[6] J. Jiang, K. A. Alderisio, A. Singh, and L. Xiao, "Development of procedures for direct extraction of Cryptosporidium DNA from water concentrates and for relief of PCR inhibitors," Applied and Environmental Microbiology, vol. 71, no. 3, pp. 1135-1141, 2005.

[7] M. W. Ware, L. Wymer, H. D. A. Lindquist, and F. W. Schaefer III, "Evaluation of an alternative IMS dissociation procedure for use with Method 1622: detection of Cryptosporidium in water," Journal of Microbiological Methods, vol. 55, no. 3, pp. 575-583, 2003.

[8] L. Xiao, A. Singh, and K. Alderisio, Development and Standardization of a Cryptosporidium Genotyping Tool for Water Samples, AWWA Research Foundation, Denver, Colo, USA, 2006.

[9] Y. Ochiai, C. Takada, and M. Hosaka, "Detection and discrimination of Cryptosporidium parvum and C. hominis in water 
samples by immunomagnetic separation-PCR," Applied and Environmental Microbiology, vol. 71, no. 2, pp. 898-903, 2005.

[10] United States Environmental Protection Agency, "Method 1623Cryptosporidium and Giardia in water by filtration/IMS/FA," U.S. Environmental Protection Agency publication no. 821/R01-026, United States Environmental Protection Agency, Washington, DC, USA, 2001.

[11] R. A. Guy, P. Payment, U. J. Krull, and P. A. Horgen, "Realtime PCR for quantification of Giardia and Cryptosporidium in environmental water samples and sewage," Applied and Environmental Microbiology, vol. 69, no. 9, pp. 5178-5185, 2003.

[12] J. J. Verweij, R. ten Hove, E. A. T. Brienen, and L. van Lieshout, "Multiplex detection of Enterocytozoon bieneusi and Encephalitozoon spp. in fecal samples using real-time PCR," Diagnostic Microbiology and Infectious Disease, vol. 57, no. 2, pp. 163-167, 2007.

[13] D. M. Wolk, S. K. Schneider, N. L. Wengenack, L. M. Sloan, and J. E. Rosenblatt, "Real-time PCR method for detection of Encephalitozoon intestinalis from stool specimens," Journal of Clinical Microbiology, vol. 40, no. 11, pp. 3922-3928, 2002.

[14] P. G. Hartel, "Minimizing microbial source tracking at all costs," in Microbial Source Tracking: Methods, Applications, and Case Studies, C. Hagedorn, A. Blanche, and V. J. Harwood, Eds., Springer, New York, NY, USA, 2011.

[15] J. A. Moss and R. A. Snyder, "Protozoan pathogens," in Microbial Source Tracking: Methods, Applications, and Case Studies, C. Hagedorn, A. Blanche, and V. J. Harwood, Eds., Springer, New York, NY, USA, 2011.

[16] U. S. Environmental Protection Agency, "Microbial source tracking guide document," Tech. Rep. EPA/600-R-05-064, Office of Research and Development, Cincinnati, Ohio, USA, 2005.

[17] U. S. Environmental Protection Agency, Source Water Monitoring Guidance Manual for Public Water Systems for the Long Term 2 Enhanced Surface Water Treatment Rule (LT2 Rule), U.S. Environmental Protection Agency publication no. 815/D-03005, U.S. Environmental Protection Agency, Washington, DC, USA, 2003.

[18] J. Jiang, K. A. Alderisio, L. Singh, and L. Xiao, "Distribution of Cryptosporidium genotypes in storm event water samples from three watersheds in New York," Applied and Environmental Microbiology, vol. 71, no. 8, pp. 4446-4454, 2005.

[19] L. Xiao, K. Alderisio, J. Limor, M. Royer, and A. A. Lal, "Identification of species and sources of Cryptosporidium oocysts in storm waters with a small-subunit rRNA-based diagnostic and genotyping tool," Applied and Environmental Microbiology, vol. 66, no. 12, pp. 5492-5498, 2000.

[20] L. Xiao, K. A. Alderisio, and J. Jiang, "Detection of Cryptosporidium oocysts in water: effect of the number of samples and analytic replicates on test results," Applied and Environmental Microbiology, vol. 72, no. 9, pp. 5942-5947, 2006.

[21] T. Wohlsen, J. Bates, B. Gray, and M. Katouli, "Evaluation of five membrane filtration methods for recovery of Cryptosporidium and Giardia isolates from water samples," Applied and Environmental Microbiology, vol. 70, no. 4, pp. 2318-2322, 2004.

[22] J. E. Aldom and A. H. Chagla, "Recovery of Cryptosporidium oocysts from water by a membrane filter dissolution method," Letters in Applied Microbiology, vol. 20, no. 3, pp. 186-187, 1995.

[23] J. E. Aldom and A. H. Chagla, "Membrane filter dissolution: a novel method for the recovery of Cryptosporidium oocysts from water," in Proceedings of the International Symposium on Waterborne Cryptosporidium Proceedings, American Water Works Association, C. R. Fricker, J. L. Clancy, and P. A. Rochelle, Eds., pp. 87-89, Denver, Colo, USA, 1997.

[24] R. M. McCuin, Z. Bukhari, and J. L. Clancy, "Recovery and viability of Cryptosporidium parvum oocysts and Giardia intestinalis cysts using the membrane dissolution procedure," Canadian Journal of Microbiology, vol. 46, no. 8, pp. 700-707, 2000.

[25] R. Briancesco, E. Veschetti, M. Ottaviani, and L. Bonadonna, "Peracetic acid and sodium hypochlorite effectiveness in reducing resistant stages of microorganisms," Central European Journal of Public Health, vol. 13, no. 3, pp. 159-162, 2005.

[26] A. F. Maheux, L. Bissonnette, M. Boissinot et al., "Method for rapid and sensitive detection of Enterococcus sp. and Enterococcus faecalis/faecium cells in potable water samples," Water Research, vol. 45, no. 6, pp. 2342-2354, 2011.

[27] M. Bednarska, A. Bajer, E. Sinski, A. S. Girouard, L. Tamang, and T. K. Graczyk, "Fluorescent in situ hybridization as a tool to retrospectively identify Cryptosporidium parvum and Giardia lamblia in samples from terrestrial mammalian wildlife," Parasitology Research, vol. 100, no. 3, pp. 455-460, 2007.

[28] S. M. Cacciò, "New methods for the diagnosis of Cryptosporidium e Giardia," Parassitologia, vol. 46, no. 1-2, pp. 151-155, 2004.

[29] T. K. Graczyk, R. Fayer, M. R. Cranfield, and R. Owens, "Cryptosporidium parvum oocysts recovered from water by the membrane filter dissolution method retain their infectivity," Journal of Parasitology, vol. 83, no. 1, pp. 111-114, 1997.

[30] C. C. Brescia, S. M. Griffin, M. W. Ware, E. A. Varughese, A. I. Egorov, and E. N. Villegas, "Cryptosporidium propidium monoazide-PCR, a molecular biology-based technique for genotyping of viable Cryptosporidium oocysts," Applied and Environmental Microbiology, vol. 75, no. 21, pp. 6856-6863, 2009.

[31] R. C. Rendtorff, “The experimental transmission of human intestinal protozoan parasites: II. Giardia lamblia cysts gwen in capsules," The American Journal of Epidemiology, vol. 59, no. 2, pp. 209-220, 1954.

[32] R. C. Rendtorff, "The experimental transmission of Giardia lamblia among volunteer subjects," in Waterborne Transmission of Giardiasis, W. Jakubowski and J. C. Hoff, Eds., EPA-600/979-001, pp. 64-81, U.S. Environmental Protection Agency, Cincinnati, Ohio, USA, 1979.

[33] H. L. DuPont, C. L. Chappell, C. R. Sterling, P. C. Okhuysen, J. B. Rose, and W. Jakubowski, "The infectivity of Cryptosporidium parvum in healthy volunteers," New England Journal of Medicine, vol. 332, no. 13, pp. 855-859, 1995.

[34] P. C. Okhuysen, C. L. Chappell, J. H. Crabb, C. R. Sterling, and H. L. DuPont, "Virulence of three distinct Cryptosporidium parvum isolates for healthy adults," Journal of Infectious Diseases, vol. 180, no. 4, pp. 1275-1281, 1999.

[35] T. L. Heitman, L. M. Frederick, J. R. Viste et al., "Prevalence of Giardia and Cryptosporidium and characterization of Cryptosporidium spp. isolated from wildlife, human, and agricultural sources in the North Saskatchewan River Basin in Alberta, Canada," Canadian Journal of Microbiology, vol. 48, no. 6, pp. 530-541, 2002.

[36] S. Coupe, K. Delabre, R. Pouillot, S. Houdart, M. SantillanaHayat, and F. Derouin, "Detection of Cryptosporidium, Giardia and Enterocytozoon bieneusi in surface water, including recreational areas: a one-year prospective study," FEMS Immunology and Medical Microbiology, vol. 47, no. 3, pp. 351-359, 2006. 
[37] W. Dang, "The swimmer exposure assessment model (SWIMODEL) and its use in estimating risks of chemical use in swimming pool," EPA Internal Guidance Document, US Environmental Protection Agency, Washington, DC, USA, 1996.

[38] M. Santín and R. Fayer, "Microsporidiosis: enterocytozoon bieneusi in domesticated and wild animals," Research in Veterinary Science, vol. 90, no. 3, pp. 363-371, 2011.

[39] O. Matos, M. L. Lobo, and L. Xiao, "Epidemiology of Enterocytozoon bieneusi infection in humans," Journal of Parasitology Research, vol. 2012, Article ID 981424, 19 pages, 2012.

[40] L. Xhou, A. Singh, J. Jiang, and L. Xiao, "Molecular surveillance of Cryptosporidium spp. in raw wastewater in Milwaukee: implications for understanding outbreak occurrence and transmission dynamics," Journal of Clinical Microbiology, vol. 41, no. 11, pp. 5254-5257, 2003.

[41] J. A. Higgins, R. Fayer, J. M. Trout et al., "Real-time PCR for the detection of Cryptosporidium parvum," Journal of Microbiological Methods, vol. 47, no. 3, pp. 323-337, 2001.

[42] C. F. L. Amar, P. H. Dear, and J. McLauchlin, "Detection and genotyping by real-time PCR/RFLP analyses of Giardia duodenalis from human faeces," Journal of Medical Microbiology, vol. 52, no. 8, pp. 681-683, 2003. 


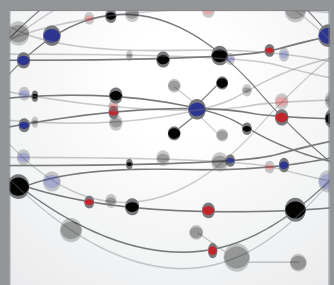

The Scientific World Journal
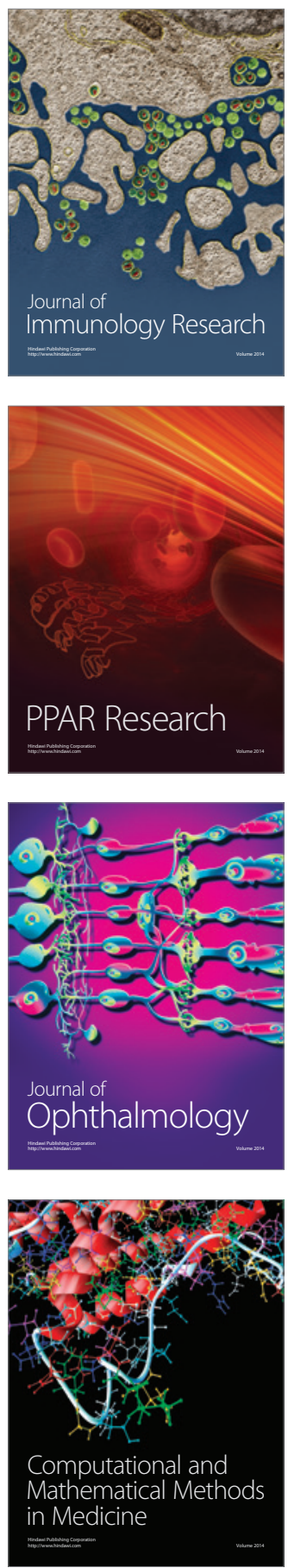

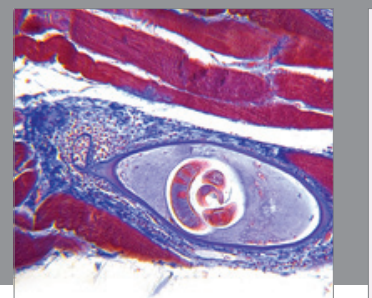

Gastroenterology

Research and Practice
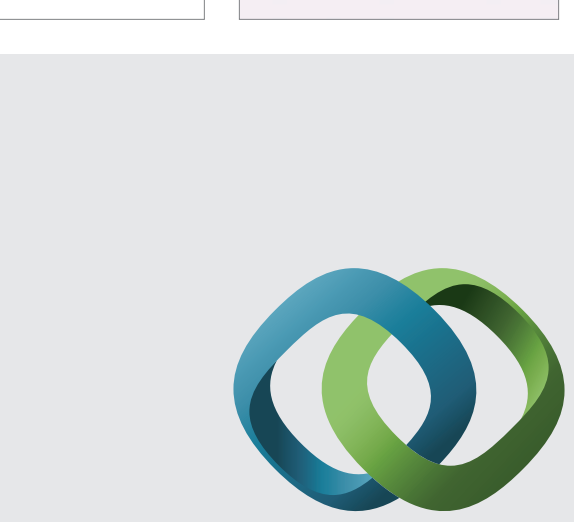

\section{Hindawi}

Submit your manuscripts at

http://www.hindawi.com
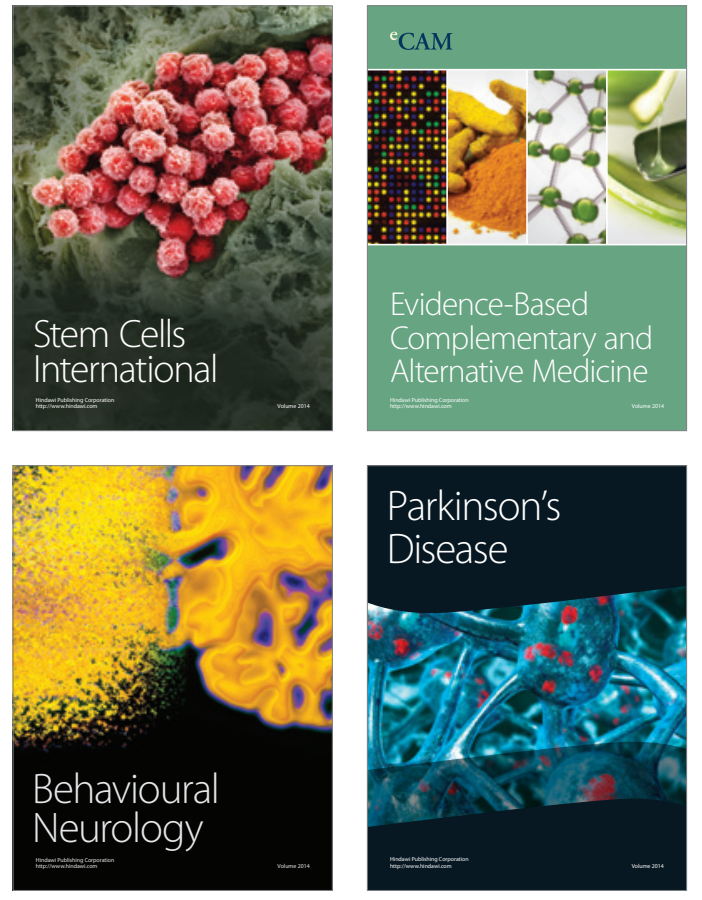
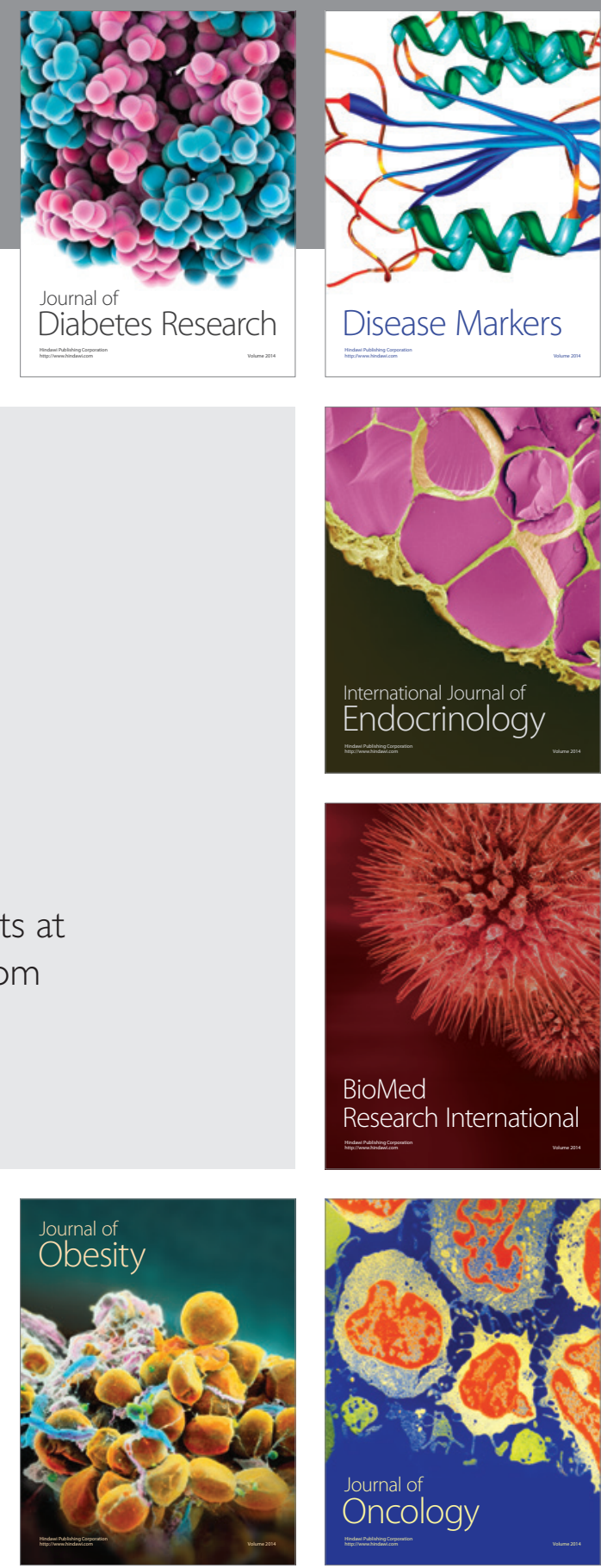

Disease Markers
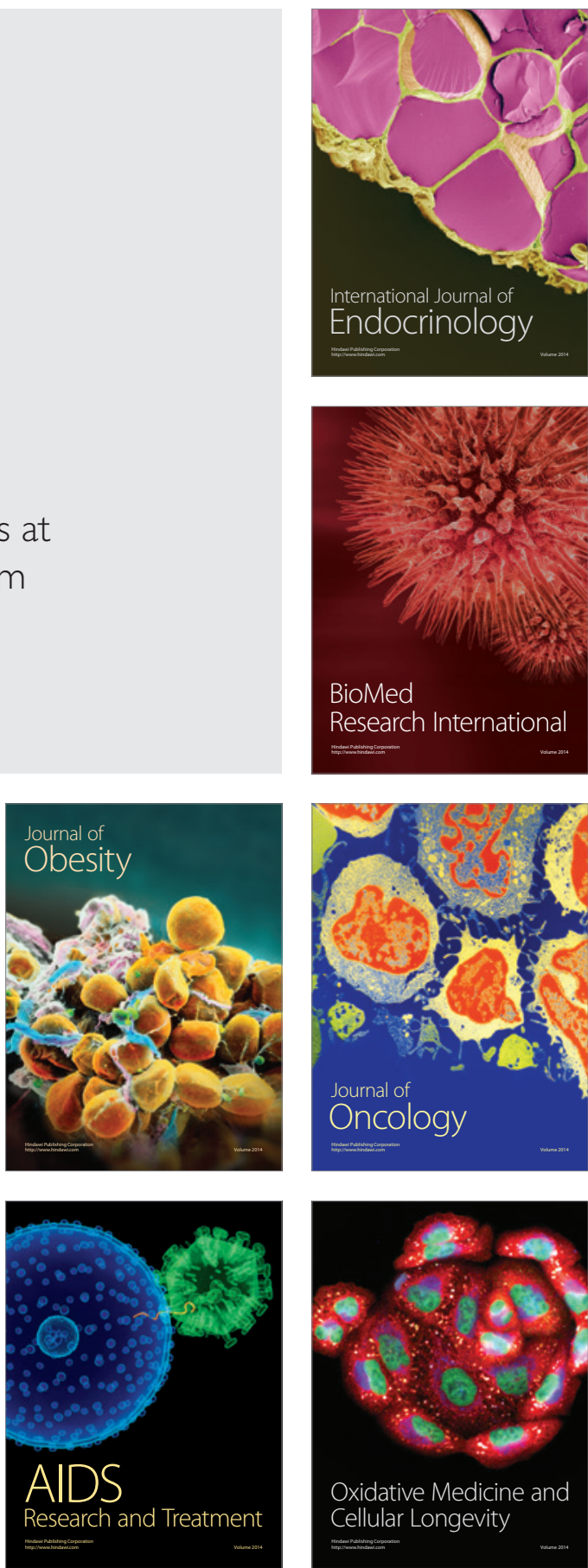